\title{
Tratamiento endoscópico del dolor en los pacientes con pancreatitis crónica
}

\author{
D. LEDRO CANO, J. M. CATALÁN RAMÍREZ, M. J. GONZÁLEZ MARISCAL, \\ R. ROMERO CASTRO, F. J. PELLICER BAUTISTA, J. M. HERRERÍAS \\ Unidad de Endoscopia. Servicio de Aparato Digestivo. Área Hospitalaria Virgen \\ Macarena. Sevilla
}

\author{
ENDOSCOPIC PAIN MANAGEMENT IN CHRONIC PANCREATITIS \\ PATIENTS
}

\begin{abstract}
RESUMEN
Introducción: La Pancreatitis crónica es una enfermedad recurrente, frecuentemente asociada al consumo de alcohol y suele presentarse en la tercera y la cuarta décadas. Las estenosis y las litiasis del conducto pancreático principal producen obstrucción del mismo y consecuentemente dolor. Por tanto la descompresión quirúrgica o endoscópica, del conducto pancreático principal alivia el dolor.

Sujetos y métodos: Revisión de nuestra experiencia en el manejo endoscópico en pacientes con pancreatitis crónica. Realizamos CPREs en 42 pacientes para el manejo de su pancreatitis crónica. Las opciones terapéuticas fueron la esfinterotomía, colocación de endoprótesis ó ninguna. El seguimiento medio fue 47,8 $(27,2)$ meses.

Resultados: 22 pacientes fueron sometidos a CPRE terapéutica. En ocho pacientes, realizamos esfinterotomía y en 14 pacientes, colocamos endoprótesis con esfinterotomía previa. 8 pacientes estaban asintomáticos, después de un seguimiento medio de 10,8 (11,6) meses. Dos de ellos, fueron sometidos a una esfinterotomía y en seis de ellos, colocamos endoprótesis, previa esfinterotomía.

Conclusión: La CPRE es una herramienta que alivia el dolor en un quinto de pacientes con pancreatitis crónica.
\end{abstract}

PALABRAS CLAVE: Dolor. Pancreatitis Crónica. CPRE. Prótesis. Esfinterotomía.

\begin{abstract}
Introduction: Chronic Pancreatitis is a recurrent disease, frequently alcohol intake related and tend to occur in the third and the fourth decades. Stenoses and lithiasis in the main pancreatic duct causes obs truction and subsequently pain. Therefore endoscopic or surgical decompression of main pancreatic duct results in pain relief.

Subjects and methods: Review our experience in the endoscopic management in patients suffering from chronic pancreatitis. 42 patients underwent ERCP for management of their chronic pancreatitis. Thera peutic options were sphincterotomy alone, prostheses and "do nothing". Follow-up was made by phone call and outpatient office visits. Mean follow-up was 47.8 (27.2) months.

Results: 22 patients underwent therapeutic ERCP. In 8 patients we performed sphincterotomy and in 14 patients, a sphincterotomy and prostheses intubation. 8 patients are asymptomatic after a mean followup of 10.8 (11.6) months. 2 of them, underwent sphincterotomy and six of them, underwent sphincterotomy and prostheses intubation.

Conclusion: Therapeutic ERCP is a tool that relieves pain in a fifth of patients suffering from chronic pancreatitis.
\end{abstract}

KEY WORDS: Pain. Chronic Pancreatitis. ERCP. Prostheses. Sphincte rotomy.

Ledro Cano D, Catalán Ramírez JM, González Mariscal MJ, Romero Castro R, Pellicer Bautista FJ, Herrerías JM. Tratamiento endoscópico del dolor en los pacientes con pancreatitis crónica. An Med Interna (Madrid) 2002; 19: 69-72.

\section{INTRODUCCIÓN}

La Pancreatitis crónica (PC) fue descrita por Comfort y cols. (1) como una enfermedad recidivante, frecuentemente asociada al consumo de alcohol, especialmente en la tercera y cuarta década de la vida con complicaciones asociadas. El alcohol podría actuar como tóxico sobre las células acinares, provocando acúmulos de lípidos en dichas células (2) y favoreciendo el paso de enzimas proteolíticos al torrente sanguíneo (3). Por otra parte el alcohol aumentaría la secreción pancreática, la secreción proteica y alteraría la composición del jugo pancreático (disminuye la secreción de bicarbonato), favoreciendo la precipitación de proteínas y la formación de tapones de células ductales, calcio cristalizado y matriz proteica cristalizada (4). El consumo reiterado de alcohol podría dañar la barrera pancreática, permitiendo la retrodifusión de enzimas pancreáticas activadas al parénquima circundante (5) y de la misma manera pudiera modificar el gradiente de presión bilio-pancreático-duodenal e influir en las condiciones de flujo-reflujo (6). La obstrucción del conducto pancreático principal está ocasionada por la presencia de litiasis y/o de estenosis $(7,8)$. Como consecuencia de todo ello, el paciente presenta dolor (9), esteatorrea así como el desarrollo de un cuadro de diabetes mellitus secundaria con la consiguiente pérdida de peso.

Trabajo aceptado: 31 de octubre de 2001

Correspondencia: Diego Ledro Cano. Virgen de Luján, $455^{\circ} \mathrm{C}$. Sevilla 41011. e-mail: dledro@ supercable.es 
Ésta es la razón fundamental de la necesidad de técnicas de drenaje, las cuales pueden ser quirúrgicas o endoscópicas $(10,11)$. Algunos de estos grupos de trabajo ha realizado seguimiento de sus pacientes hasta durante cuatro años (12).

Revisaremos la experiencia de nuestro servicio en el manejo endoscópico de los pacientes con pancreatitis crónica desde Enero del año 1993 hasta Noviembre del año 2000. Los pacientes fueron remitidos a nuestro servicio por la presencia de dolor abdominal rebelde a analgésicos mayores. En ellos evaluamos la efectividad de la técnica en términos de alivio del dolor, el tiempo de la recidiva del dolor y/o recambio de la prótesis, la necesidad de cirugía y la presencia de complicaciones.

\section{MATERIAL Y MÉTODOS}

Entre enero del año 1993 hasta noviembre del año 2000, 42 pacientes fueron sometidos a una Colangiopancreatografía Retrógrada Endoscópica (C.P.R.E.) por sospecha diagnóstica de PC, por necesidad de tratamiento endoscópico o bien por seguimiento de su PC. En 3 pacientes no se pudo realizar la exploración $(7 \%)$, en otros tres pacientes, la pancreatografía era normal y estaban asintomáticos, por lo que se decidió no realizar terapéutica alguna. De los 36 pacientes restantes con pancreatografía anormal y presencia de síntomas, en 22 de ellos realizamos terapéutica $(61,1 \%)$.

\section{CRITERIOS DE INCLUSIÓN Y DE EXCLUSIÓN}

Se considero criterio de inclusión para tratamiento endoscópico la presencia de estenosis única o múltiple con dilatación segmentaria del conducto de Wirsung con o sin litiasis intraductal. Los criterios de exclusión fueron pseudoquiste no comunicado al conducto pancreático principal o la ausencia de consentimiento informado. Otros criterios de exclusión fueron el abandono del seguimiento por parte del paciente y la imposibilidad y/o la falta de indicación de realizar terapéutica.

\section{PROCEDIMIENTO}

El procedimiento endoscópico, diagnóstico y terapéutico se realizaba en el mismo acto médico, previa sedoanalgesia con diacepam y petidina, empleándose como espasmolítico butilbromuro de hioscina. Empleamos un videoduodenoscopio con canal de trabajo de 4,2 $\mathrm{mm}$, catéteres estándar de colangiografía, así como otros con modificaciones técnicas en su extremo distal para facilitar la canulación (punta roma, punta fina), guías metálicas de diversos calibres (0,035 a 0,018 pulgadas), con extremo distal recto o curvo y empleo de manipuladores de guía. En caso de encontrar una estenosis del conducto de Wirsung inferior a $1 \mathrm{~mm}$ de calibre, aplicábamos dilatadores por pulsión, montados sobre guía, con calibre máximo de 7 French como paso previo a la inserción de la prótesis. Cuando se diagnosticaba litiasis intraductal, se procedía a su extracción mediante cesta tipo Dormia. Las prótesis empleadas son de material plástico (teflón, modelo Solopas) con orificio de drenaje en ambos extremos, con calibre externo de 7 French y longitud variable entre 5 y $10 \mathrm{cms}$. El procedimiento empleado en la inserción de prótesis consistía en realizar canulación selectiva del conducto pancreático principal, papilotomía y esfinterotomia selectiva del conducto de Wirsung. A continuación y una vez estudiada la morfología del conducto de Wirsung mediante inyección de contraste yodado en su interior (presencia o no de cálculos, localización y número de estenosis), se procedía a la dilatación de le estenosis, previa colocación de la guía a través de las mismas. En caso de litiasis, se llevaba a cabo su extracción y finalmente insertábamos la prótesis, salvando la zona estenosada, a través de la guía fijadora mencionada. En el caso de no poder, colocar una guía a través de la estenosis, simplemente realizábamos una esfinterotomia endoscópica selectiva del conducto de Wirsung.

\section{SEGUIMIENTO}

La eficacia del tratamiento se evaluó como la desaparición del dolor. Los cambios de las prótesis se consideraron en aquellos pacientes en los que recurrió el dolor. El tiempo de supervivencia de la prótesis se considero como el período libre de dolor, desde la inserción de la prótesis hasta su recambio. Estos datos fueron recopilados mediante la entrevista personal en las revisiones en las consultas externas, llamadas telefónicas y revisión de las historias clínicas.

Del total de pacientes sometidos a dicha técnica en 22 realizamos terapéutica $(52,38 \%)$. La edad media de los sujetos fue $51,37(14,88)$ años. La distribución por sexos fue la siguiente 3 Mujeres $(13,63 \%)$ y 19 hombres (6,36\%). El número medio de C.P.R.E.s por paciente fue de $2,5(2,4)$ veces y el seguimiento medio fue de $47,8(27,2)$ meses.

\section{RESULTADOS}

De los 22 pacientes sometidos a C.P.R.E. terapéutica, en 8 de ellos, exclusivamente realizamos esfinterotomía endoscópica y en los 14 restantes, realizamos esfinterotomía endoscópica seguida de colocación de endoprotesis. 8 pacientes están asintomáticos tras un seguimiento medio de 10,8 (11,6) meses, lo que supone un $36,36 \%$ de los pacientes tratados (por protocolo) y un $19,04 \%$ de los pacientes de nuestra serie (por intención de tratar). 5 pacientes requirieron cirugía $(22,7 \%)$, de los cuales 4 para control sintomático y uno de ellos, para el tratamiento quirúrgico de un absceso.

-Pacientes tratados sólo con Esfinterotomía Endoscópica.

- 2 pacientes se encuentran asintomáticos (25\%), 2 pacientes presentan dolor $(25 \%), 2$ pacientes requirieron cirugía (25\%) y 2 pacientes fallecieron por carcinoma pancreático (25\%).

-Pacientes tratados con Esfinterotomía Endoscópica y colocación de Endoprótesis.

- 6 pacientes están asintomáticos (42,85\%) (Tabla I), 1 paciente presenta dolor $(7,14 \%), 1$ paciente ha fallecido por carcinoma pancreático $(7,14 \%), 3$ pacientes abandonaron el seguimiento por pertenecer a otra área sanitaria $(21,4 \%)$ y 3 pacientes han sido sometidos a cirugía $(21,4 \%)$ (2 de ellos están asintomáticos tras drenaje con la técnica de Puestow y uno de ellos, fue intervenido por pseudoquiste complicado). 
TABLA

\begin{tabular}{|c|c|c|c|c|}
\hline \multicolumn{5}{|c|}{$\begin{array}{l}\text { SEGUIM IENTO DE PACIENTES CON PANCREATITIS } \\
\text { CRÓ NICA, TRATADO S CON ESFINTEROTO MÍA } \\
\text { ENDO SCOO PICA Y COLO CACIÓN DE PRÓ TESIS }\end{array}$} \\
\hline Número & Wirsung & $\begin{array}{l}\text { Número de } \\
\text { Stents }\end{array}$ & $\begin{array}{c}\text { Duración } \\
\text { (días) }\end{array}$ & $\begin{array}{l}\text { Longitud } \\
\text { (cms) }\end{array}$ \\
\hline 1 & $\begin{array}{l}\text { Estenosis } \\
\text { no proximal }\end{array}$ & 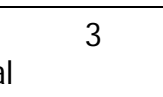 & $450 ; 900 ; 120$ & $5 ; 10 ; 7$ \\
\hline 2 & $\begin{array}{l}\text { Estenosis y } \\
\text { dilataciones } \\
\text { con cálculos }\end{array}$ & s & 60 & 7 \\
\hline 3 & $\begin{array}{l}\text { Estenosis } \\
\text { proximal }\end{array}$ & 2 & $90 ; 60$ & $5 ; 10$ \\
\hline 4 & $\begin{array}{l}\text { Estenosis y } \\
\text { dilataciones } \\
\text { con cálculos }\end{array}$ & s & 390 & 7 \\
\hline 5 & $\begin{array}{l}\text { Estenosis no } \\
\text { proximal }\end{array}$ & 0 & $60 ; 1980$ & 5 \\
\hline 6 & $\begin{array}{l}\text { Estenosis y } \\
\text { dilataciones } \\
\text { con cálculos }\end{array}$ & s & 1620 & 5 \\
\hline
\end{tabular}

(Imagen del W irsung. Número de Stents. Duración de los Stents y Longitud de los Stents)

La duración media de cada endoprotesis fue de 10,8 $(11,6)$ meses y el número medio de recambios por paciente fue de $1,08(1,5)$ meses.

\section{DISCUSIÓN}

Nuestra tasa de sujetos de $61,1 \%$, en los cuáles pudimos realizar endoscopia terapéutica es inferior a las comunicadas por el grupo de Hammarstrom, donde alcanzan el 70\% (13) y por el estudio de Smits donde se alcanzaba el 77\% (14).

Los pacientes tratados con esfinterotomía endoscópica junto con colocación de prótesis representaban un $63,6 \%$ de los pacientes sometidos a terapéutica, cifra sensiblemente superior a la del grupo sueco, previamente mencionado que era del $46,1 \%$ (13).

Nuestro período de seguimiento medio fue de $47,8(27,2)$ meses. Este período es superior al referido por Cremer y cols. que era de 37 meses así como en el caso de Smits y cols. que fue de 33 meses $(15,13)$. Otro grupo procedente de la República Checa, muestra un seguimiento de 24 meses (16). A idéntico seguimiento fueron sometidos los pacientes del grupo de Dumonceau y cols. (17). Aunque algunos autores como Soehendra, realizaron un seguimiento medio de 58,8 meses (12). Nuestro período es muy similar a los 44 meses, comunicados por un grupo Japonés (18) y al de 48 meses de Hammarstrom y cols. (14). Un estudio reciente, en pacientes sometidos a esfinterotomia endoscópica exclusivamente, el seguimiento fue de 16 meses (19).
La necesidad de cirugía en nuestra serie fue $22,7 \%$, un resultado muy similar al grupo de Cremer que combinó drenaje pancreático con litotripsia extracorpórea, donde tenían un $15 \%$ de necesidad de tratamiento quirúrgico (15). Este mismo grupo, propone un recambio de la prótesis a los 12 meses, tiempo muy parecido a la duración de nuestras prótesis, que fue de 10,8 $(11,6)$ meses. Otro grupo alemán, recambiaba las prótesis a una media de 15,7 meses, durante un seguimiento de casi cuatro años (12).

En nuestro estudio, el porcentaje de pacientes sometidos a terapéutica endoscópica y que permanecían asintomáticos al final del estudio era $36,3 \%$, superior al $9,7 \%$ comunicado por Delhaye y cols. (20) que utilizaban litotripsia extracorpórea y drenaje pancreático, con un seguimiento de 12 meses. Binmoeller y cols. siguen durante cinco años a un grupo de pacientes con pancreatitis crónica, tratados con drenaje endoscópico mediante prótesis, observando que hasta un 64,5\% están asintomáticos, cifra ligeramente superior al $42,8 \%$ de nuestra serie (12). Tras un año seguimiento, Ponchon y cols. que trataron a sus pacientes con estenosis distales con dilatadores y prótesis de 10 French, observaron una mejoría del dolor del 50\% de los pacientes al año de seguimiento (21).

En pacientes con pancreatitis crónica con litiasis intraductal, un estudio realizado en Amsterdam, muestra como el $71,6 \%$ de los pacientes están asintomáticos, tras la extracción de los cálculos y el drenaje pancreático, tras un seguimiento de 33 meses. En esta serie, el 15\% de los sujetos requirieron drenaje quirúrgico (13).

En nuestra serie, el 25\% de los sujetos con pancreatitis crónica, tratados sólo con esfinterotomía endoscópica, permanecían asintomáticos al final de un seguimiento medio de 11 meses, estos resultados son inferiores a los presentados por el grupo de Dite, donde se alcanza una tasa de alivio del dolor cercana al a los dos años de seguimiento (16), si bien en nuestra serie de ocho sujetos, dos pacientes fueron sometidos a cirugía, un paciente abandono el seguimiento y otro paciente falleció.

Ohara y cols. señalan que con el uso de la litotripsia extracorpórea junto con una esfinterotomía endoscópica, se alcanza una mejoría del $80 \%$ a los 44 meses de seguimiento (18).

El 60\% de los pacientes sometidos a esfinterotomía endoscópica, después de un seguimiento de 16 meses, se encontraban asintomáticos, según publican Okolo y cols. en un estudio reciente (19).

Hammarstrom y cols. intentaron tratar a 18 pacientes con pancreatitis crónica, obteniendo un drenaje pancreático en 13 de ellos, mediante esfinterotomia endoscópica en 7 pacientes y mediante esfinterotomía endoscópica con colocación de prótesis en 6 pacientes. Un $62 \%$ de los pacientes tratados se encuentran asintomáticos a los 2 años, dicha cifra es superior al 36,3\% de nuestro estudio. 3 pacientes necesitaron cirugía, lo que representa el $23 \%$ de dicha serie (14), muy similar a nuestros resultados del $22,7 \%$.

En nuestro país son ya varios los grupos que trabajan con éxito en el campo de la terapia endoscópica de la pancreatitis crónica, siendo pionero el grupo de la Dra. Salas que aplica en dos pacientes litotricia extracorpórea y colocación de endoprótesis pancreática en sendos pacientes con pancreatitis crónica calcificante (22).

Dumonceau realizando esfinterotomía endoscópica y extracción de cálculos intraductuales, comunica un porcentaje de pacientes con alivio de su dolor cercano al 54\%, al final de los dos años de seguimiento, estos datos sugieren que el dolor 
de la pancreatitis crónica pudiera ser debido a un aumento de la presión intraductal (17). Basándonos en esta hipótesis, ya recogida en estudios anteriores $(7,8)$, desde el año 1993, nuestro grupo comenzó a profundizar en el tratamiento endoscópico del dolor en los pacientes con pancreatitis crónica, nuestras primeras experiencias dieron como fruto la publicación de un serie de seis pacientes afectados de pancreatitis crónica enólica, tratados mediante colocación de prótesis y con un seguimiento medio de 7 meses, donde pudimos concluir que el drenaje pancreático endoscópico mediante endoprótesis es eficaz como tratamiento del dolor. Es un procedimiento seguro sin complicaciones. Logrando colocar la prótesis a través de la estenosis, podemos aliviar el dolor (23).

\section{CONCLUSIONES}

La terapéutica endoscópica es una técnica efectiva y segura. Debe realizarse en centros experimentados con un volumen elevado de casos. La terapéutica necesita disponer de dispositivos auxiliares como fluoroscopia de alta definición. El seguimiento de los pacientes debe ser adecuado, mediante las revisiones periódicas en las consultas externas. Debe existir una estrecha colaboración con radiólogos, cirujanos y anatomopatólogos expertos. El tratamiento debe ser variable, adaptándose a los problemas sucesivos que ocurren a lo largo del curso de esta enfermedad crónica.

\section{Bibliografía}

1. Comfort MW, Gambil EE, Baggenstoss AH. Chronic relapsing pancreatitis. A study of twenty-nine cases without associated disease of the biliary or gastrointestinal tract. Gastroenterology 1946; 6: 239-285.

2. Noronha M, Dreiling DA, Bordalo O. Sequential changes from minimal pancreatic inflammation to advanced alcoholic pamcreatitis. Z Gastroenterol 1983; 21: 666-673

3. Hayakawa T, Noda A, Kondo T. Changes in serum pancreatic enzyme during 2 months abstinence in asymptomatic chronic alcoholics . Am J Gastroenterol 1982; 77: 625-629.

4. Sahel J, Sarles H. Modification of pure human pancreatic juice induced by chronic alcohol consumption. Dig Dis sci 1979; 24: 897-905.

5. Nagata A, Homma T, Kama K, et al. A study of chronic pancreatitis by serial endoscopic pancreatography. Gastroenterology 1981; 81 : 884-891.

6. Izsak EM, Shike M, Romlet M, et al. Pancreatitis in association with hypercalcemia in patients receiving total parenteral nutrition. Gastroenterology 1980; 79: 555-558.

7. Bradley EL. Pancreatic duct pressure in chronic pancreatitis. Am J Surg 1982; 144: 303-316.

8. Ebbehoj N, Borly L, Bulow J, et al. Pancreatic tissue fluid pressure in chronic pancreatitis: relation to pain, morphology and function. Scand $\mathbf{J}$ Gastroenterol 1990; 25: 1046-1051.

9. Layer P, Yakamoto H, Kalthoff L, Clain JE, Bakken LJ, Di Margno EP. The different courses of early and late onset idiopathic and alcoholic pancreatitis. Gastroenterology 1994; 107: 1481-1487.

10. Prinz RA, Greenlee HB. Pancreatic duct drainage in chronic pancreatitis. Hepato-Gatroenterol 1990; 37: 295-300.

11. Sarles JC, Nacchiero M, Garani F, Salasc B. Surgical treatment of chronic pancreatitis: report of 134 cases treated by resection or drainage. Am J Surg 1982; 144: 317-321.

12. Binmoeller KF, Jue P, Seifert H, Nam WC, Izbicki J, Soehendra M. Endoscopic pancreatic stent drainage in chronic pancreatitis and a dominant stricture: long- term results. Endoscopy 1995; 27: 638-644.

13. Smits ME, Rauws EA, Tytgat GN, Huibregtse K. Endoscopic treatment of pancreatic stones in patients with chronic pancreatitis. Gastrointest Endosc 1996; 43(6): 556-560.

14. Hammarstrom LE, Stridbeck H, Ihse I. Endoscopic drainage in benign pancreatic disease: immediate and medium term outcome. Eur J Surg 1997; 163(8): 577-589.

15. Cremer M, Deviere J, Delhaye M, Baize M, Vandermeeren A. Stenting in severe chronic pancreatitis: results of medium-term follow-up in seventy-six patients. Endoscopy 1991; 23(3): 171-176.

16. Dite P, Zboril V, Cikankova E. Endoscopic therapy of chronic pancreatitis. Hepatogastroenterology 1996; 43(12): 1633-1637.

17. Dumonceau JM, Deviere J, Le Moine O, Delhaye M, Vandermeeren A, Baize M, et al. Endoscopic pancreatic drainage in chronic pancreatitis associated with ductal stones: long-term results. Gastrointest Endosc 1996; 4(6): 547-555.

18. Ohara H, Hoshino M, Hayakawa T, Kamiya Y, Miyaji M, Takeuchi T, et al. Single application extracorporeal shock wave lithotripsy is the first choice for patients with pancreatic duct stones. Am J Gastroenterol 1996; 91(7): 1388-1394.

19. Okolo PI III, Pasricha PJ, Kalloo AN. What are the long-term results of endoscopic pancreatic sphincterotomy? Gastrointest Endosc 2000; 52(1): 15-19.

20. Delhaye M, Vandermeeren A, Baize M, Cremer M. Extracorporeal shock-wave lithotripsy of pancreatic calculi. Gastroenterology 1992; 102(2): 610-620.

21. Ponchon T, Bory RM, Hedelius F, Roubein LD, Paliard P, Napoleon B, et al. Endoscopic stenting for pain relief in chronic pancreatitis : results of a standardized protocol. Gastrointest Endosc 1995; 42(5): 452-456.

22. Sala T, Pertejo V, Berenguer M, Higon M, Boronat F, Berenguer J. Tratamiento endoscópico combinado con litotricia extracorpórea en la pancreatitis crónica calcificante. Rev Esp Enferm Dig 1994; 86: 596-599.

23. Pellicer Bautista FJ, Fernández Pérez FJ, Martín Guerrero JM, Pallarés Manrique H, Hassan Asad M, Romero Castro R, et al. Tratamiento del dolor en la pancreatitis crónica mediante prótesis pancreáticas. Rev Esp Enferm Dig 1998; 90(1): 23-27. 